\title{
Many-particle interference to test Born's rule
}

\author{
Marc-Oliver Pleinert $\odot,{ }^{1,2}$ Joachim von Zanthier, ${ }^{1,2}$ and Eric Lutz ${ }^{3}$ \\ ${ }^{1}$ Institut für Optik, Information und Photonik, Friedrich-Alexander-Universität Erlangen-Nürnberg (FAU), D-91058 Erlangen, Germany \\ ${ }^{2}$ Erlangen Graduate School in Advanced Optical Technologies (SAOT), Friedrich-Alexander-Universität Erlangen-Nürnberg (FAU), \\ D-91052 Erlangen, Germany \\ ${ }^{3}$ Institute for Theoretical Physics I, University of Stuttgart, D-70550 Stuttgart, Germany
}

(Received 19 October 2018; revised manuscript received 12 November 2019; accepted 10 February 2020; published 28 February 2020)

\begin{abstract}
Born's rule relates detection probabilities to the modulus square of the wave function. It is one of the central principles of quantum mechanics together with that of linear superposition. Single-particle interference is accordingly limited to pairs of quantum paths and higher-order interferences are prohibited. Deviations from Born's law have been quantified via the Sorkin parameter which is proportional to the third-order term. However, while the linearity of quantum theory has been experimentally tested to the level of $10^{-20} \mathrm{eV}$, the Sorkin parameter has only been measured to an accuracy of $2 \times 10^{-3}$ in the quantum regime. We here investigate Born's law using many-particle interferences and demonstrate that all interference terms of order $(2 M+1)$ and greater vanish for $M$ particles. We further introduce a family of many-particle Sorkin parameters and show that they are exponentially more sensitive to deviations from Born's rule than their single-particle counterpart.
\end{abstract}

DOI: 10.1103/PhysRevResearch.2.012051

No deviation from the predictions of quantum theory has been observed so far. Yet, there is no reason why quantum mechanics should be an ultimate theory. High-precision tests of quantum mechanics itself (as opposed to derived theories, such as nuclear or particle physics) are therefore invaluable to explore its limits [1-4]. While it is difficult to find logically consistent generalizations of quantum physics [5], various fundamental concepts of the quantum formalism have been extensively examined experimentally in past decades [6,7], especially its linearity [8-14]. Nonlinear corrections to quantum mechanics have been shown to be smaller than $1.5 \times$ $10^{-20} \mathrm{eV}$ [13]. However, despite its importance, experimental studies of Born's rule have only been undertaken recently [15-20].

Born's rule states that the probability of a measurement outcome is given by the modulus square of the corresponding probability amplitude [21]. This fundamental postulate of quantum mechanics establishes a link between the (deterministic) mathematical formalism and experiment. It additionally introduces a random component into the theory. In view of its significance, many attempts to replace the postulate by a derivation from underlying principles have been made [22-28]. Consequences of a deviation from the rule have been theoretically explored [29-32] and consistent modifications have been constructed for single and bipartite systems [30-32]. However, to date these derivations are still subject to

Published by the American Physical Society under the terms of the Creative Commons Attribution 4.0 International license. Further distribution of this work must maintain attribution to the author(s) and the published article's title, journal citation, and DOI. discussion [33-37]. Experimental tests of Born's law are thus crucial to assess its accuracy.

A direct consequence of Born's rule is that single-particle quantum interference originates only from pairs of quantum paths [38]. Interferences of higher order than the second therefore do not occur in quantum mechanics for single particles. The vanishing of third-order interference can be quantified with the help of the so-called Sorkin parameter [see Eq. (3) below], which is zero if Born's rule holds [38]. The parameter has been measured in three- and five-slit experiments with single photons [15-17] and single molecules [18,19], and has been found to be smaller than $3 \times 10^{-5}$ in the classical light regime and $2 \times 10^{-3}$ in the quantum regime [17]. These findings rule out higher-order single-particle interference [39] and confirm Born's law to that level of precision.

Quantum mechanics, however, is not limited to singleparticle interference phenomena. In the case of indistinguishable particles, it also allows for many-particle interference, which is mathematically richer and physically more subtle than single-particle interference $[40,41]$. For example, two photons, albeit noninteracting, can influence each other via two-particle interference as in the Hong-Ou-Mandel experiment [42]. Here, two indistinguishable photons impinging on a 50:50 beam splitter will not exit the output ports of the beam splitter separately due to the destructive interference of the two different two-photon paths, where both photons are transmitted or reflected, respectively. Many-particle interference has been observed in a variety of bosonic and fermionic systems, including photons [42-50], phonons [51], and bosonic atoms [52,53], as well as fermionic atoms [53-55] and electrons [56-58]. It is not only of interest from a fundamental point of view [40-46,59], but has also been exploited in imaging $[47,48]$, metrology $[49,60]$, and for quantum information processing $[50,61]$. 
We here propose to use many-particle interference for a fundamental test of Born's rule, and thus of quantum theory. We study general setups with arbitrary numbers of particles, where many-particle interferences show up in the higher-order spatial correlations among the particles. Using Glauber's theory of quantum coherence and higher-order correlations [62], we derive higher-order sum rules that allow us to test Born's rule and introduce a family of many-particle Sorkin parameters. In particular, we demonstrate that all interference terms of order $(2 M+1)$ and larger vanish for $M$ particles. We further show that the generalized Sorkin parameters are exponentially more sensitive with increasing $M$ to deviations from Born's rule than their single-particle counterpart.

Single-particle interference. In the standard double-slit experiment, interference can be quantified by comparing the pattern $P_{A B}(\mathbf{r}, t)$ observed when both slits $A$ and $B$ are open to the classical expectation of the sum of two single slits, $P_{A}(\mathbf{r}, t)+P_{B}(\mathbf{r}, t)$, where either slit $A$ or slit $B$ is open [63]. Here, $P(\mathbf{r}, t)$ stands for the detection probability of a single particle at position $\mathbf{r}$ and time $t$. The interference term is thus given by

$$
I^{(1)}:=P_{A B}-\left(P_{A}+P_{B}\right),
$$

where we indicate in the superscript that we are dealing with single-particle interference [and omit the arguments $(\mathbf{r}, t)$ for simplicity] [64]. Equation (1) can be computed quantum mechanically by applying Born's rule (BR) and the superposition principle (SP) [65],

$$
\begin{aligned}
P_{A B} & \stackrel{(\text { BR })}{=}\left|\psi_{A B}\right|^{2} \stackrel{(\text { SP })}{=}\left|\psi_{A}+\psi_{B}\right|^{2} \\
& =\left|\psi_{A}\right|^{2}+\left|\psi_{B}\right|^{2}+\psi_{A}^{*} \psi_{B}+\psi_{B}^{*} \psi_{A} \\
& =P_{A}+P_{B}+I^{(1)},
\end{aligned}
$$

where $\psi_{X}$ denotes the wave function of a particle at the detection plane when the slit configuration $X=A, B, A B$ is open. Any nonzero value of $I^{(1)}$ indicates a deviation from the ordinary (incoherent) sum of the subsystems $A$ and $B$, and thus any interference present between those. In experiments with particles whose wave functions are spatially coherent over the slits, $I^{(1)}$ typically shows a sinusoidal behavior [63].

In classical physics (with distinguishable particles), the interference term $I^{(1)}$ is zero, yielding the classical additivity of mutually exclusive events. By contrast, the formalism of quantum mechanics predicts a nonzero second-order interference term $I^{(1)} \equiv I_{2}^{(1)} \neq 0$, while all higher-order interference terms $I_{N}^{(1)}(N \geqslant 3)$ vanish [38]. The usual interference term $I_{2}^{(1)}$ emerges naturally in double-slit experiments and marks off classical from quantum mechanics. On the other hand, the third-order interference term $I_{3}^{(1)}$ turning up in triple-slit setups allows one to distinguish quantum mechanics from generalized probabilistic theories. This quantity is also called the (single-particle) Sorkin parameter $\epsilon$, defined as

$$
\begin{aligned}
\epsilon(\mathbf{r}) & \equiv I_{3}^{(1)}(\mathbf{r}) \\
& :=P_{A B C}-\left(P_{A B}+P_{A C}+P_{B C}\right)+\left(P_{A}+P_{B}+P_{C}\right) .
\end{aligned}
$$

The pattern $P_{A B C}$ observed after a triple slit can again be derived by applying BR and SP [38]. It turns out that all occurring interference terms are already included in the double-slit terms. The triple-slit pattern can hence be written as a sum of the comprised double and single slits and no genuine thirdorder interference term survives, $I_{3}^{(1)}=0$. This is commonly referred to as the nonexistence of higher-order single-particle interference $[15,38]$. Equation (3) can be used to quantify deviations from Born's rule which predicts $\epsilon=0$. Experiments often introduce a normalized Sorkin parameter $\kappa=\epsilon / P_{\text {norm }}$, where $P_{\text {norm }}$ is the sum of second-order interferences [15], the total number of counts [18], or the central maximum of the triple slit [20]. We will here consider the form of Eq. (3).

Many-particle interference. In the case of many-particle phenomena, it is convenient to work within the second quantization framework, where the tedious symmetrization of wave functions is inherent in the operators. This will lead us to work with correlation functions. The $M$ th-order intensity correlation function is defined by [62]

$$
G^{(M)}\left(\mathbf{r}_{1}, t_{1}, \ldots, \mathbf{r}_{M}, t_{M}\right)=\left\langle\hat{a}_{1}^{\dagger} \cdots \hat{a}_{M}^{\dagger} \hat{a}_{M} \cdots \hat{a}_{1}\right\rangle,
$$

where $\hat{a}_{i}=\hat{a}\left(\mathbf{r}_{i}, t_{i}\right)$ is the bosonic annihilation operator at the position $\mathbf{r}_{i}$ of the $i$ th detector and time of detection $t_{i}$. Evaluated for an $M$-particle wave function, $G^{(M)}$ equals the joint probability to detect $M$ photons at positions $\mathbf{r}_{1}, \ldots, \mathbf{r}_{M}$ and times $t_{1}, \ldots, t_{M}$, that is, a correlated detection at different detectors as shown in Fig. 1(a). Throughout this study, we will concentrate on coincident events, $t_{i}=t$, and omit the time (and position) dependence for simplicity. We note that the first-order correlation function $G_{X}^{(1)}$ is equal to the detection probability $P_{X}$ for any slit combination $X$, when evaluated for a single-particle wave function $\psi_{X}$. Hence, all single-particle interference results can be analyzed in terms of the first-order correlation function $G^{(1)}$

In order to examine many-particle interference, we assume, as in the case of single-particle interference, that the wave function of the $M$ particles is spatially coherent over the separation of the slits. At first, we note that for a single slit $A$, we have $G_{A}^{(M)}=\prod_{i=1}^{M} G_{A}^{(1)}\left(\mathbf{r}_{i}\right) \equiv \prod_{i=1}^{M} G_{A, \mathrm{cl} .}^{(1)}\left(\mathbf{r}_{i}\right)=G_{A, \mathrm{cl} .}^{(M)}$, since there is no interference in single-slit experiments even when using quantum particles. The first configuration to exhibit (many-particle) interference is the double slit. To trigger a coincident event at $M$ detectors behind a double slit, there are in total $2^{M}$ distinct $M$-particle paths. Classically, all these events are mutually exclusive and the classical signal is simply given by the incoherent sum of the corresponding contributions,

$$
G_{A B, \mathrm{cl} .}^{(M)}=G_{A}^{(M)}+\sum_{j=1}^{M} G_{A}^{(M-1)}\left(\left\{\mathbf{r}_{i \neq j}\right\}_{i=1, \ldots, M}\right) G_{B}^{(1)}\left(\mathbf{r}_{j}\right)+\cdots .
$$

Here, the first term represents the event, in which all detected particles came from $A$, the second expression includes all paths, in which one of the detected particles was from $B$ and $M-1$ from $A$, and so on. The usual interference term, i.e., here the second-order $M$-particle interference, is defined in analogy to Eq. (1) as

$$
I_{2}^{(M)}:=G_{A B}^{(M)}-G_{A B, \mathrm{cl} .}^{(M)},
$$

where $G_{A B}^{(M)}$ is the quantum-mechanical result of Eq. (4) including the interference between all occurring $M$-particle paths. 

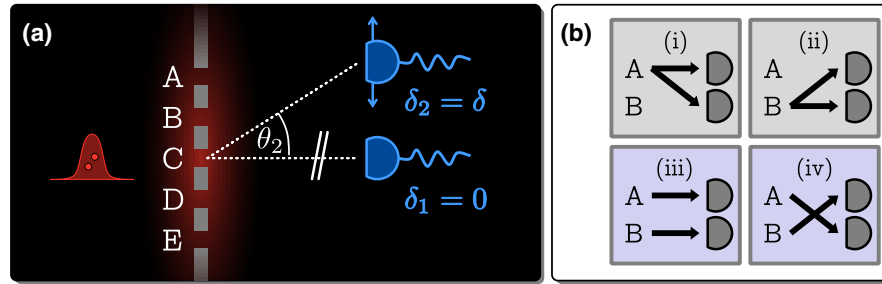

(c)

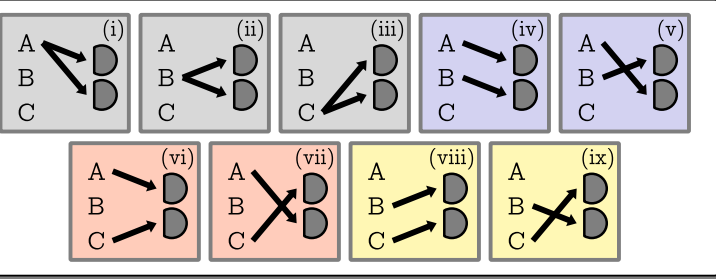

(d)

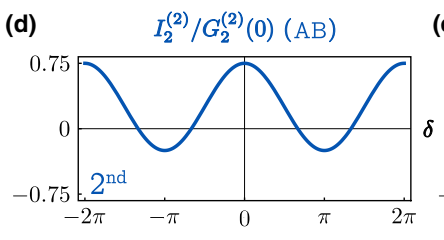

(e) $\quad I_{3}^{(2)} / G_{3}^{(2)}(0)(\mathrm{ABC})$

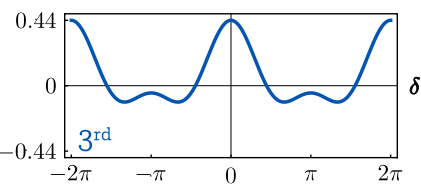

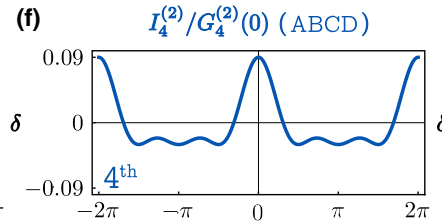

(g) $\quad I_{5}^{(2)}(\mathrm{ABCDE})$

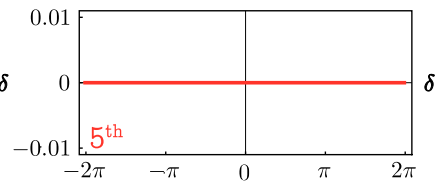

FIG. 1. Two-particle interference in slit experiments with up to five slits. (a) Sketch of the fivefold slit and the detection configuration: One detector is fixed $\left(\delta_{1}=0\right)$ while the other one is scanned $\left(\delta_{2}=\delta\right)$. The coincident detection signal arises (b) in a double-slit setup from four and (c) in a triple-slit setup from nine mutually exclusive two-particle paths which can interfere. (d)-(g) Theoretically evaluated second- to fifth-order two-particle interference, Eq. (7) (normalized to the central maximum of the related slit pattern), as a function of the optical phase $\delta$ for the detection technique indicated in (a).

We can now generalize this second-order many-particle interference term to an arbitrary order of interference. In doing so, it becomes apparent that we can assign a great number of occurring terms (but not all) to either arising from classical terms or from interferences of lower order [66]. We can thus define a hierarchy of higher-order many-particle interference terms containing the particular interference of $N$ th order that was not present in any lower-order many-particle interference. In this way, the $N$ th-order $M$-particle interference can be written as [66]

$$
\begin{aligned}
I_{N}^{(M)}:= & G_{A_{1}, A_{2}, \ldots, A_{N}}^{(M)}-\mathcal{G}_{A_{1}, A_{2}, \ldots, A_{N}, \mathrm{cl} .}^{(M)} \\
& +\sum_{l=1}^{N-1}(-1)^{l} \sum_{\sigma \in \mathcal{C}_{N-l}^{N}} \underbrace{(M)}_{A_{\sigma_{1}}, A_{\sigma_{2}}, \ldots, A_{\sigma_{N-l}}} .
\end{aligned}
$$

Equation (7) is straightforward to comprehend by considering interference patterns from $N$-slit experiments: The first term represents the quantum-mechanical signal from $N$ slits [see Eq. (4)], from which we subtract the classical $M$-particle paths $\mathcal{G}_{A_{1}, A_{2}, \ldots, A_{N}, \text { cl. }}^{(M)}$ arising from exactly $N$ different slits [66]. The expression in the second line of Eq. (7) subtracts from $G_{A_{1}, A_{2}, \ldots, A_{N}}^{(M)}$ the contributions to the signal of $N$ slits from lower-order terms (including interference from all comprised $k$-slit configurations with $k<N$ ), where $\mathcal{C}_{k}^{N}$ stands for the set of all $k$-combinations of $\{1,2, \ldots, N\}$. Note that Eq. (7) is defined such that it is also valid for the single-particle case $M=$ 1 [see Eq. (1)]. The formal resemblance of single- and manyparticle interference hierarchies originates from a similar packing of the occurring terms. However, they differ strongly. The second-order two-particle interference $I_{2}^{(2)}$, for instance, is composed of up to 12 single terms compared to only 2 for the second-order single-particle interference $I_{2}^{(1)}$ [66].

While the third-order single-particle interference term is the first to vanish in the family of single-particle interference terms, many-particle interference yields a much richer structure. For example, for two-particle interference $(M=2)$ after a double slit $(N=2)$ there are $N^{M}=2^{2}=4$ different two-particle paths labeling the different options how two indistinguishable particles can reach two detectors, namely, the detected particles are either (i) both from $A$, or (ii) both from $B$, or one from $A$ and one from $B$, where (iii) detector 1 registered the particle from $A$ and detector 2 registered the particle from $B$, or (iv) detector 1 registered the particle from $B$ and detector 2 registered the particle from $A$, respectively. These paths are shown in Fig. 1(b). The classical signal can thus be written as $G_{A B, \text { cl. }}^{(2)}=G_{A}^{(2)}+G_{B}^{(2)}+G_{A}^{(1)}\left(\mathbf{r}_{1}\right) G_{B}^{(1)}\left(\mathbf{r}_{2}\right)+$ $G_{A}^{(1)}\left(\mathbf{r}_{2}\right) G_{B}^{(1)}\left(\mathbf{r}_{1}\right)$, and the second-order two-particle interference is given by $I_{2}^{(2)}=G_{A B}^{(2)}-G_{A B \text {,cl. }}^{(2)}$, which is nonzero [66]. Considering the next order $I_{3}^{(2)}$, it turns out that two-particle interference in a triple-slit-experiment can, unlike the singleparticle analog, not be decomposed into a sum of lower-order two-particle interference patterns, even though all interfering terms shown in Fig. 1(c) do already appear in the appropriate lower-order ones [67]. Third-order two-particle interference hence does not vanish, which holds also true for the fourthorder: $I_{3}^{(2)} \neq 0$ and $I_{4}^{(2)} \neq 0$ [66]. The fifth-order two-particle interference, however, is now the first of the family members to vanish $I_{5}^{(2)}=0$, and thus all $I_{N}^{(2)}=0$ for $N \geqslant 5$.

For this two-particle case, we have theoretically evaluated the two-particle interference terms of up to fifth order for the detector configuration shown in Fig. 1(a). In this setup, $(M-1)$ detectors are located at fixed positions, while the $M$ th detector scans the detection plane. This technique is commonly used to explore many-particle phenomena, such as superradiance [45] and subradiance [46]. It is also used for quantum imaging [48]. Our findings for $M=2$ are shown in Figs. 1(d)-1(g). Here, the respective interferences are plotted as a function of the optical phases $\delta_{i}\left(\mathbf{r}_{i}\right)=2 \pi d \sin \left(\theta_{i}\right) / \lambda$ involving the slit distance $d$, the angle of detection $\theta_{i}$ of the $i$ th detector [see Fig. 1(a)], and the wavelength $\lambda$. For the nonzero orders, we normalize the interferences to the maximum of the slit pattern to which they contribute, e.g., for the second order $(A B): I_{2}^{(2)} / G_{A B}^{(2)}(0)$. We note that $I_{5}^{(2)}=0$ [see Fig. $\left.1(\mathrm{~g})\right]$ is independent of the actual detector configuration [66].

We stress that nonvanishing third- and fourth-order twoparticle interference is in line with Born's rule, which in this 
context predicts that quantum interference originates from pairs of two-particle paths, which is why fifth-order twoparticle interference has to vanish. Generally speaking, Born's law predicts that quantum interferences arise from pairs of $M$-particle paths, i.e., up to order $2 M$. As a result, by recursion all interferences of order $(2 M+1)$ and larger are zero for $M$ particles.

Accordingly, we can now introduce a family of Sorkin parameters for $M$ particles,

$$
\epsilon^{(M)}\left(\mathbf{r}_{1}, \ldots, \mathbf{r}_{M}\right):=I_{2 M+1}^{(M)}\left(\mathbf{r}_{1}, \ldots, \mathbf{r}_{M}\right),
$$

where the first member $(M=1)$ is the original Sorkin parameter of Eq. (3). Born's law predicts that all these parameters vanish regardless of the detector positions.

Sensitivity to deviations. We next establish that the manyparticle Sorkin parameters are significantly more sensitive to deviations from Born's rule than the corresponding singleparticle parameter. To this end, we consider a small deviation from Born's rule such that the probability of an event $X$ is given by $P_{X}=\left|\psi_{X}\right|^{2+\eta}$ with $|\eta| \ll 1$. Such deviations directly lead to nonzero many-particle Sorkin parameters $\epsilon_{\eta}^{(M)}$.

To be concrete, we evaluate the parameters $\epsilon_{\eta}^{(M)}$ for the detection configuration described above and outlined for $M=2$ in Fig. 1(a). We choose the fixed positions, $\mathbf{r}_{i}, i=1, \ldots, M-$ 1 , of the $(M-1)$ detectors such that the first detector is on the optical axis [see Fig. 1(a)] and the remaining $(M-2)$ fixed detectors are located such that the optical phase difference accumulated by particles originating from the same slit but propagating to different detectors is a multiple of $2 \pi$. This can, for instance, be achieved with $\delta_{i}\left(\mathbf{r}_{i}\right)=(i-1) 2 \pi, i=$ $1, \ldots, M-1$. Limiting ourselves to the $M$-particle Sorkin parameter at $\delta_{M}=0$, we can then relate the $M$ th-order correlation function $G^{(M)}$ to the $M$ th power of the central peak of the first-order correlation function $\left[G^{(1)}(0)\right]^{M}[66]$. This allows us to compare the magnitude of the Sorkin parameters for different particle numbers $M$ for the same deviation $\eta$. For $|\eta| \ll 1$, we obtain [66]

$$
\epsilon_{\eta}^{(M)}(0)=\sum_{k=1}^{2 M+1}(-1)^{k-1}\left(\begin{array}{c}
2 M+1 \\
k
\end{array}\right) M k^{2 M} \log (k) \eta .
$$

The sum accounts for all subslit configurations contributing with magnitude $M k^{2 M} \log (k)$ to the Sorkin parameter induced by a nonzero $\eta$. Equation (9) vanishes for $\eta=0$, as it should, and expresses the sensitivity to deviations $\eta$ from Born's rule for $M$-particle interference.

Figure 2(a) shows the exact absolute sensitivity to deviations of the many-particle Sorkin parameter (symbols), as well as the analytical formula $\epsilon_{\eta}^{(M)}(0)$ [Eq. (9)] (lines) in the small $\eta$ limit, as a function of the particle number $M$ for three different values of $\eta$. We observe that the magnitude of $\epsilon_{\eta}^{(1)}$ in the single-particle case $(M=1)$ is roughly determined by the order of the deviation $\eta$ itself. Yet, the absolute sensitivity to deviations from Born's rule is significantly improved, by several orders of magnitude, with increasing values of $M$ for many-particle interference. Moreover, while the absolute sensitivity vanishes in the limit $\eta \rightarrow 0$ for all $M$, the relative sensitivity, defined as the ratio $\epsilon_{\eta}^{(M)}(0) / \epsilon_{\eta}^{(1)}(0)$, is independent of $\eta$ and thus has a finite value for $\eta \rightarrow 0$ [66]. Figure 2(b) displays the relative sensitivity as a function of $M$ for the
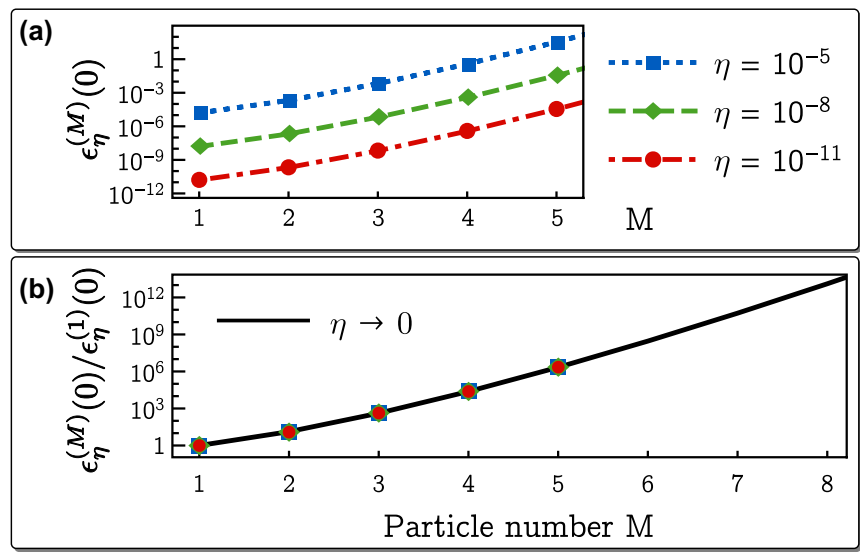

FIG. 2. Exponential increase in sensitivity of the $M$-particle Sorkin parameter to a deviation from Born's rule of the general form $\left|\psi_{X}\right|^{2+\eta}$. (a) Absolute sensitivity $\epsilon_{\eta}^{(M)}(0)$ for three values of $\eta$ : numerical values (symbols) and analytical approximation, Eq. (9) (lines). (b) Relative sensitivity $\epsilon_{\eta}^{(M)}(0) / \epsilon_{\eta}^{(1)}(0)$ : numerical values [symbols as in (a)] and analytical approximation, Eq. (9) in the limit $\eta \rightarrow 0$ (solid line).

numerical simulation (symbols) and the analytical formula (solid line) in the limit $\eta \rightarrow 0$. Remarkably, the increase depends exponentially on the number of particles, linked to the exponential increase in the number of interfering paths. Any nonzero deviation from Born's rule of the form $\left|\psi_{X}\right|^{2+\eta}$, no matter how tiny, will therefore be drastically enhanced in the case of the many-particle Sorkin parameters, by a factor of about 13 for the discussed two-particle example, by two orders of magnitude for three-particle, and 13 orders of magnitude for eight-particle interference. The level of manyparticle interference is not limited in principle. Eight-particle interference has, for instance, already been measured for superradiant emission [45].

Other effects may lead to nonzero Sorkin parameters. However, these are either very small or can be distinguished from violations of Born's rule. For instance, deviations originating from nonlinear corrections have been shown to be smaller than $1.5 \times 10^{-20} \mathrm{eV}$ [13]. Additional departures coming from nonclassical looped paths $[20,68,69]$ can be bounded by $10^{-22}$ in an interferometer with macroscopic distances between the different paths [17]. Moreover, deviations from Born's law should be independent of specific interferometer configurations, such as number of slits or distance from the slits.

Conclusion. We have demonstrated that many-particle interference can substantially increase the sensitivity in experimental tests of Born's rule. Using Glauber's theory of quantum coherence, we have established that for $M$-particle interference all interference terms of order $(2 M+1)$ and larger vanish if Born's rule holds. Accordingly, the generalized Sorkin parameters, derived for many-particle interference, are shown to be zero independent of the detection configuration. Yet, the generalized $M$-particle Sorkin parameters have the benefit of displaying an exponentially increased sensitivity to deviations from Born's rule with respect to their single-particle counterpart. We thus expect them to stimulate theoretical and experimental studies, as well as more precise tests of quantum theory. 
Acknowledgments. M.-O.P. gratefully acknowledges financial support by the Studienstiftung des deutschen Volkes. M.-O.P. and J.v.Z. gratefully acknowledge funding by the
Erlangen Graduate School in Advanced Optical Technologies (SAOT) by the German Research Foundation (DFG) in the framework of the German excellence initiative.
[1] S. Weinberg, Testing quantum mechanics, Ann. Phys. (NY) 194, 336 (1989).

[2] S. Weinberg, Precision Tests of Quantum Mechanics, Phys. Rev. Lett. 62, 485 (1989).

[3] I. Bialynicki-Birula and J. Mycielski, Nonlinear wave mechanics, Ann. Phys. 100, 62 (1976).

[4] A. Shimony, Proposed neutron interferometer test of some nonlinear variants of wave mechanics, Phys. Rev. A 20, 394 (1979).

[5] S. Aaronson, Is quantum mechanics an island in theoryspace? arXiv:quant-ph/0401062.

[6] A. Bassi, K. Lochan, S. Satin, T. P. Singh, and H. Ulbricht, Models of wave-function collapse, underlying theories, and experimental tests, Rev. Mod. Phys. 85, 471 (2013).

[7] M. Arndt and K. Hornberger, Testing the limits of quantum mechanical superpositions, Nat. Phys. 10, 271 (2014).

[8] C. G. Shull, D. K. Atwood, J. Arthur, and M. A. Horne, Search for a Nonlinear Variant of the Schrödinger Equation by Neutron Interferometry, Phys. Rev. Lett. 44, 765 (1980).

[9] R. Gähler, A. G. Klein, and A. Zeilinger, Neutron optical tests of nonlinear wave mechanics, Phys. Rev. A 23, 1611 (1981).

[10] J. J. Bollinger, D. J. Heinzen, W. M. Itano, S. L. Gilbert, and D. J. Wineland, Test of the Linearity of Quantum Mechanics by rf Spectroscopy of the ${ }^{9} \mathrm{Be}^{+}$Ground State, Phys. Rev. Lett. 63, 1031 (1989).

[11] T. E. Chupp and R. J. Hoare, Coherence in Freely Precessing ${ }^{21} \mathrm{Ne}$ and a Test of Linearity of Quantum Mechanics, Phys. Rev. Lett. 64, 2261 (1990).

[12] R. L. Walsworth, I. F. Silvera, E. M. Mattison, and R. F. C. Vessot, Test of the Linearity of Quantum Mechanics in an Atomic System with a Hydrogen Maser, Phys. Rev. Lett. 64, 2599 (1990).

[13] P. K. Majumder, B. J. Venema, S. K. Lamoreaux, B. R. Heckel, and E. N. Fortson, Test of the Linearity of Quantum Mechanics in Optically Pumped ${ }^{201} \mathrm{Hg}$, Phys. Rev. Lett. 65, 2931 (1990).

[14] A. Vinante, R. Mezzena, P. Falferi, M. Carlesso, and A. Bassi, Improved Noninterferometric Test of Collapse Models using Ultracold Cantilevers, Phys. Rev. Lett. 119, 110401 (2017).

[15] U. Sinha, C. Couteau, T. Jennewein, R. Laflamme, and G. Weihs, Ruling out multi-order interference in quantum mechanics, Science 329, 418 (2010).

[16] I. Söllner, B. Gschösser, P. Mai, B. Pressl, Z. Vörös, and G. Weihs, Testing Born's rule in quantum mechanics for three mutually exclusive events, Found. Phys. 42, 742 (2012).

[17] T. Kauten, R. Keil, T. Kaufmann, B. Pressl, Č. Brukner, and G. Weihs, Obtaining tight bounds on higher-order interferences with a 5-path interferometer, New J. Phys. 19, 033017 (2017).

[18] J. P. Cotter, C. Brand, C. Knobloch, Yi. Lilach, O. Cheshnovsky, and M. Arndt, In search of multipath interference using large molecules, Sci. Adv. 3, e1602478 (2017).

[19] A. R. Barnea, O. Cheshnovsky, and U. Even, Matter-wave diffraction approaching limits predicted by Feynman path in- tegrals for multipath interference, Phys. Rev. A 97, 023601 (2018).

[20] O. S. Magaña-Loaiza, I. De Leon, M. Mirhosseini, R. Fickler, A. Safari, U. Mick, B. McIntyre, P. Banzer, B. Rodenburg, G. Leuchs, and R. W. Boyd, Exotic looped trajectories of photons in three-slit interference, Nat. Commun. 7, 13987 (2016).

[21] M. Born, Zur Quantenmechanik der Stoßvorgänge, Z. Phys. 37, 863 (1926).

[22] H. Everett, "Relative state" formulation of quantum mechanics, Rev. Mod. Phys. 29, 454 (1957).

[23] J. B. Hartle, Quantum mechanics of individual systems, Am. J. Phys. 36, 704 (1968).

[24] E. Farhi, J. Goldstone, and S. Gutmann, How probability arises in quantum mechanics, Ann. Phys. (NY) 192, 368 (1989).

[25] D. Deutsch, Quantum theory of probability and decisions, Proc. R. Soc. London, Ser. A 455, 3129 (1999).

[26] W. H. Zurek, Environment-Assisted Invariance, Entanglement, and Probabilities in Quantum Physics, Phys. Rev. Lett. 90, 120404 (2003).

[27] S. Saunders, Derivation of the Born rule from operational assumptions, Proc. R. Soc. London, Ser. A 460, 1771 (2004).

[28] L. Masanes, T. D. Galley, and M. P. Müller, The measurement postulates of quantum mechanics are operationally redundant, Nat. Commun. 10, 1361 (2019).

[29] W. Son, Consistent theory for causal non-locality beyond the Born's rule, J. Korean Phys. Soc. 64, 499 (2014).

[30] T. D. Galley and L. Masanes, Classification of all alternatives to the Born rule in terms of informational properties, Quantum 1, 15 (2017).

[31] T. D. Galley and L. Masanes, Any modification of the Born rule leads to a violation of the purification and local tomography principles, Quantum 2, 104 (2018).

[32] T. Galley, Modifying the measurement postulates of quantum theory, Ph.D. thesis, University College London, 2018.

[33] E. J. Squires, On an alleged "proof" of the quantum probability law, Phys. Lett. A 145, 67 (1990).

[34] A. Cassinello and J. L. Sánchez-Gómez, On the probabilistic postulate of quantum mechanics, Found. Phys. 26, 1357 (1996).

[35] H. Barnum, C. M. Caves, J. Finkelstein, C. A. Fuchs, and R. Schack, Quantum probability from decision theory? Proc. R. Soc. London, Ser. A 456, 1175 (2000).

[36] M. Schlosshauer and A. Fine, On Zurek's derivation of the Born rule, Found. Phys. 35, 197 (2005).

[37] L. Vaidman, Derivations of the Born rule, http://philsci-archive. pitt.edu/15943/.

[38] R. D. Sorkin, Quantum mechanics as quantum measure theory, Mod. Phys. Lett. A 09, 3119 (1994).

[39] C. M. Lee and J. H. Selby, Higher-order interference in extensions of quantum theory, Found. Phys. 47, 89 (2017).

[40] J.-W. Pan, Z.-B. Chen, C.-Y. Lu, H. Weinfurter, A. Zeilinger, and M. Żukowski, Multiphoton entanglement and interferometry, Rev. Mod. Phys. 84, 777 (2012). 
[41] M. C. Tichy, Interference of identical particles from entanglement to boson-sampling, J. Phys. B: At., Mol. Opt. Phys. 47, 103001 (2014).

[42] C. K. Hong, Z. Y. Ou, and L. Mandel, Measurement of Subpicosecond Time Intervals Between Two Photons by Interference, Phys. Rev. Lett. 59, 2044 (1987).

[43] S. Agne, T. Kauten, J. Jin, E. Meyer-Scott, J. Z. Salvail, D. R. Hamel, K. J. Resch, G. Weihs, and T. Jennewein, Observation of Genuine Three-Photon Interference, Phys. Rev. Lett. 118, 153602 (2017).

[44] A. J. Menssen, A. E. Jones, B. J. Metcalf, M. C. Tichy, S. Barz, W. S. Kolthammer, and I. A. Walmsley, Distinguishability and Many-Particle Interference, Phys. Rev. Lett. 118, 153603 (2017).

[45] S. Oppel, R. Wiegner, G. S. Agarwal, and J. von Zanthier, Directional Superradiant Emission from Statistically Independent Incoherent Nonclassical and Classical Sources, Phys. Rev. Lett. 113, 263606 (2014).

[46] D. Bhatti, R. Schneider, S. Oppel, and J. von Zanthier, Directional Dicke Subradiance with Nonclassical and Classical Light Sources, Phys. Rev. Lett. 120, 113603 (2018).

[47] R. Hanbury Brown and R. Q. Twiss, A test of a new type of stellar interferometer on Sirius, Nature (London) 178, 1046 (1956).

[48] R. Schneider, T. Mehringer, G. Mercurio, L. Wenthaus, A. Classen, G. Brenner, O. Gorobtsov, A. Benz, D. Bhatti, L. Bocklage, B. Fischer, S. Lazarev, Y. Obukhov, K. Schlage, P. Skopintsev, J. Wagner, F. Waldmann, S. Willing, I. Zaluzhnyy, W. Wurth et al., Quantum imaging with incoherently scattered light from a free-electron laser, Nat. Phys. 14, 126 (2017).

[49] Z.-E. Su, Y. Li, P. P. Rohde, H.-L. Huang, X.-L. Wang, L. Li, N.-L. Liu, J. P. Dowling, C.-Y. Lu, and J.-W. Pan, Multiphoton Interference in Quantum Fourier Transform Circuits and Applications to Quantum Metrology, Phys. Rev. Lett. 119, 080502 (2017).

[50] H. Wang, Y. He, Y.-H. Li, Z.-E. Su, B. Li, H.-L. Huang, X. Ding, M.-C. Chen, C. Liu, J. Qin, J.-P. Li, Y.-M. He, C. Schneider, M. Kamp, C.-Z. Peng, S. Höfling, C.-Y. Lu, and J.-W. Pan, Highefficiency multiphoton boson sampling, Nat. Photonics 11, 361 (2017)

[51] K. Toyoda, R. Hiji, A. Noguchi, and S. Urabe, Hong-OuMandel interference of two phonons in trapped ions, Nature (London) 527, 74 (2015).

[52] R. Lopes, A. Imanaliev, A. Aspect, M. Cheneau, D. Boiron, and C. I. Westbrook, Atomic Hong-Ou-Mandel experiment, Nature (London) 520, 66 (2015).

[53] T. Jeltes, J. M. McNamara, W. Hogervorst, W. Vassen, V. Krachmalnicoff, M. Schellekens, A. Perrin, H. Chang, D. Boiron, A. Aspect, and C. I. Westbrook, Comparison of the
Hanbury Brown-Twiss effect for bosons and fermions, Nature (London) 445, 402 (2007).

[54] T. Rom, Th. Best, D. van Oosten, U. Schneider, S. Fölling, B. Paredes, and I. Bloch, Free fermion antibunching in a degenerate atomic Fermi gas released from an optical lattice, Nature (London) 444, 733 (2006).

[55] P. M. Preiss, J. H. Becher, R. Klemt, V. Klinkhamer, A. Bergschneider, N. Defenu, and S. Jochim, High-Contrast Interference of Ultracold Fermions, Phys. Rev. Lett. 122, 143602 (2019).

[56] W. D. Oliver, J. Kim, R. C. Liu, and Y. Yamamoto, Hanbury Brown and Twiss-type experiment with electrons, Science 284, 299 (1999).

[57] M. Henny, S. Oberholzer, C. Strunk, T. Heinzel, K. Ensslin, M. Holland, and C. Schönenberger, The fermionic Hanbury Brown and Twiss experiment, Science 284, 296 (1999).

[58] H. Kiesel, A. Renz, and F. Hasselbach, Observation of Hanbury Brown-Twiss anticorrelations for free electrons, Nature (London) 418, 392 (2002).

[59] S. Mährlein, S. Oppel, R. Wiegner, and J. von Zanthier, HongOu-Mandel interference without beam splitters, J. Mod. Opt. 64, 921 (2017).

[60] V. Giovannetti, S. Lloyd, and L. Maccone, Advances in quantum metrology, Nat. Photonics 5, 222 (2011).

[61] S. Aaronson and A. Arkhipov, The computational complexity of linear optics, in Proceedings of the 43rd Annual ACM Symposium on Theory of Computing, STOC '11 (ACM, New York, 2011), p. 333.

[62] R. J. Glauber, The quantum theory of optical coherence, Phys. Rev. 130, 2529 (1963).

[63] R. P. Feynman and A. R. Hibbs, Quantum Mechanics and Path Integrals (McGraw-Hill, New York, 1965).

[64] Note that "slit" here can generally (mathematically) be seen as an orthogonal projector. The formalism is thus not restricted to slit experiments.

[65] For clarity, we neglect any normalization factors in the interference terms.

[66] See Supplemental Material at http://link.aps.org/supplemental/ 10.1103/PhysRevResearch.2.012051 for additional theory and detailed calculations.

[67] The two-particle events (i)-(iii) of Fig. 1(c) do appear in singleas well as double-slit interference, while (iv), (v), and (vi), (vii), and (viii), (ix) appear in the double slits $A B, A C$, and $B C$, respectively.

[68] H. Yabuki, Feynman path integrals in the Young double-slit experiment, Int. J. Theor. Phys. 25, 159 (1986).

[69] R. Sawant, J. Samuel, A. Sinha, S. Sinha, and U. Sinha, Nonclassical Paths in Quantum Interference Experiments, Phys. Rev. Lett. 113, 120406 (2014). 